\title{
ON THE STABLE CLASSIFICATION OF CERTAIN 4-MANIFOLDS
}

\author{
Alberto Cavicchioli, Friedrich Hegenbarth and Dušan Repovš
}

We study the s-cobordism type of closed orientable (smooth or PL) 4-manifolds with free or surface fundamental groups. We prove stable classification theorems for these classes of manifolds by using surgery theory.

\section{INTRODUCTION}

In this paper we shall study closed connected (smooth or PL) 4-manifolds with special fundamental groups as free products or surface groups. For convenience, all manifolds considered will be assumed to be orientable although our results work also in the general case, provided the first Stiefel-Whitney classes coincide. The starting point for classifying manifolds is the determination of their homotopy type. For 4manifolds having finite fundamental groups with periodic homology of period four, this was done in [12] (see also [1] and [2]). The case of a cyclic fundamental group of prime order was first treated in [23]. The homotopy type of 4-manifolds with free or surface fundamental groups was completely classified in [6] and [7] respectively. In particular, closed 4 -manifolds $M$ with a free fundamental group $\Pi_{1}(M) \cong *_{p} \mathbb{Z}$ (free product of $p$ factors $\mathbb{Z}$ ) are classified, up to homotopy, by the isomorphism class of their intersection pairings $\lambda_{M}: H_{2}(M ; \Lambda) \times H_{2}(M ; \Lambda) \rightarrow \Lambda$ over the integral group ring $\Lambda=\mathbb{Z}\left[\Pi_{1}(M)\right]$. For $\Pi_{1}(M) \cong \mathbb{Z}$, we observe that the arguments developed in [11] classify these 4-manifolds, up to TOP homeomorphism, in terms of their intersection forms over $\mathbb{Z}$.

Furthermore, it was proved in [7] that a spin connected closed 4-manifold $M$ with $\Pi_{1}(M) \cong \Pi_{1}(F), F$ a closed aspherical surface, is homotopy equivalent to a connected sum of $F \times \mathbb{S}^{2}$ with a simply-connected 4-manifold.

In this paper we shall consider the problem of when the homotopy type determines a classification of manifolds up to $s$-cobordism or up to a stable homeomorphism. We

Received 31st January, 1995

Work performed under the auspicies of the G.N.S.A.G.A. of the C.N.R. (National Research Council) of Italy and partially supported by the Ministero per la Ricerca Scientifica e Tecnologica of Italy within the projects Geometria Reale e Complessa and Topologia and by the Ministry for Science and Technology of the Republic of Slovenia Research Grant No. P1-0214-101-94.

Copyright Clearance Centre, Inc. Serial-fee code: $0004-9729 / 95 \$ \$ 2.00+0.00$. 
recall that two closed 4-manifolds $M$ and $N$ are said to be stably homeomorphic if $M \# k\left(\mathbb{S}^{2} \times \mathbb{S}^{2}\right)$ is TOP homeomorphic to $N \# \ell\left(\mathbb{S}^{2} \times \mathbb{S}^{2}\right)$ for some integers $k, \ell \geqslant 0$. It is well-known that TOP $s$-cobordant 4 -manifolds are stably homeomorphic (see for example [11, Chapters 7 and 9]).

For either $\Pi_{1} \cong *_{p} \mathbb{Z}, p \geqslant 2$, or $\Pi_{1} \cong \Pi_{1}(F), F$ a closed (orientable) surface of genus at least two, the results of [11] are not applicable since the 4-dimensional disc theorem has only been established over elementary amenable groups, the class of groups generated by the class of finite groups and $\mathbb{Z}$ by the operations of extension and increasing union (see [10]). However, the above groups are sufficient to get classifications up to TOP $s$-cobordism by using surgery theory (see $[3,11,16,18,23]$ ) and recent results proved in $[8]$ which correct some mistakes of the previous papers.

Our results can be stated as follows.

THEOREM 1. Let $M$ and $N$ be closed connected orientable (smooth) 4-manifolds with a free fundamental group $\Pi_{1} \cong *_{p} \mathbb{Z}, p \geqslant 1$. Then $M$ is simple homotopy equivalent to $N$ if and only if $M$ is TOP $s$-cobordant to $N$.

In particular, if $H_{2}(M ; \mathbb{Q})=0$ then $M$ is TOP $s$-cobordant to the connected sum $\# p\left(\mathbb{S}^{1} \times \mathbb{S}^{3}\right)$.

Theorem 1 generalises a well-known result of Wall to a nonsimply-connected case [22] and improves Theorem 2.1 of [3] for this class of manifolds. Furthermore, the case $H_{2}(M ; \mathbb{Q})=0$ recovers Theorem 2 of $[14]$ as a simple corollary.

COROLlary 2. (Hillman [14]).

Let $M$ be a closed connected orientable 4-manifold with the fundamental group $\Pi_{1}(M) \cong *_{p} \mathbb{Z}, p \geqslant 1$, and Euler characteristic $\chi(M)=2(1-p)$. Then $M$ is TOP $s$-cobordant to $\# p\left(\mathbb{S}^{1} \times \mathbb{S}^{3}\right)$.

Another partial result can be obtained as follows. Suppose that $M$ is a spin (smooth or PL) 4-manifold, that is, $w_{2}(M)=0$, where $w_{2}($.$) denotes the second$ Stiefel-Whitney class.

Then one can also define the self-intersection pairing

$$
\mu_{M}: H_{2}(M ; \Lambda) \rightarrow \frac{\Lambda}{\{\lambda-\bar{\lambda}: \lambda \in \Lambda\}},
$$

where ${ }^{-}: \Lambda \rightarrow \Lambda$ is the canonical anti-involution on $\Lambda=\mathbb{Z}\left[\Pi_{1}\right]$ (see for example [11]).

Then the triple $\left(H_{2}(M ; \Lambda), \lambda_{M}, \mu_{M}\right)$ determines an element of the $4^{\text {th }}$ Wall group $L_{4}\left({ }_{p} \mathbb{Z}\right) \cong \mathbb{Z}$ (see [4]). Under this isomorphism the class of $\left(H_{2}(M ; \Lambda), \lambda_{M}, \mu_{M}\right)$ corresponds to $(1 / 8) \operatorname{sign}(M) \in \mathbb{Z}$. Let us denote by $M^{\prime}$ the simply-connected smooth 4-manifold obtained from $M$ by killing the fundamental group. The intersection forms over $\mathbb{Z}$ of $M$ and $M^{\prime}$ are the same, as shown in [5]. Since sign $(M)=$ 
sign $\left(M^{\prime}\right)$, the definition of $L_{4}\left(\Pi_{1}\right)$ yields that the (self-)intersection pairings of $M$ and $M^{\prime} \# p\left(\mathbb{S}^{1} \times \mathbb{S}^{3}\right)$ are stably isomorphic over $\Lambda$. Thus the manifolds $M \# k\left(\mathbb{S}^{2} \times \mathbb{S}^{2}\right)$ and $M^{\prime} \# p\left(\mathbb{S}^{1} \times \mathbb{S}^{3}\right) \# \ell\left(\mathbb{S}^{2} \times \mathbb{S}^{2}\right)$ are homotopy equivalent for some $k, \ell \geqslant 0$ (see [6, Theorem 1]). Now Theorem 1 above implies that they are TOP $s$-cobordant and hence stably homeomorphic.

In summary, we have proved the following stable classification result.

ThEOREM 3. Let $M$ be a closed connected orientable (smooth or PL) 4-manifold with $w_{2}(M)=0$ and $\Pi_{1}(M) \cong *_{p} \mathbb{Z}, p \geqslant 1$. Let $M^{\prime}$ be the simply-connected manifold obtained from $M$ by killing $\Pi_{1}(M)$. Then $M$ is stably homeomorphic to $M^{\prime} \# p\left(\mathbb{S}^{1} \times \mathbb{S}^{3}\right)$.

In a special case below, one can apply the Donaldson Theorem (see [9]) to obtain the following consequence.

Corollary 4. Let $M$ be a closed connected orientable (smooth) spin 4manifold with a definite intersection form over $\mathbb{Z}$. If $\Pi_{1}(M) \cong *_{p} \mathbb{Z}, p \geqslant 1$, then $M$ is TOP s-cobordant to \#p $\left(\mathbb{S}^{1} \times \mathbb{S}^{3}\right)$.

ProOF: Let $M^{\prime}$ be as above. Then the intersection form $\lambda_{M^{\prime}}\left(\cong \lambda_{M}\right)$ is definite. The hypothesis $w_{2}(M)=0$ and $[9]$ imply that $H_{2}(M ; \mathbb{Z}) \cong H_{2}\left(M^{\prime} ; \mathbb{Z}\right) \cong 0$, hence $H_{2}(M ; \Lambda) \cong 0$ as $H_{2}(M ; \Lambda) \otimes_{\Lambda} \mathbb{Z} \cong H_{2}(M ; \mathbb{Z})$. Therefore we have $\lambda_{M} \cong 0$ over $\Lambda$ and so $M$ is simple homotopy equivalent to $\# p\left(\mathbb{S}^{1} \times \mathbb{S}^{3}\right)$ by Theorem 1 of [6]. The result now follows from Theorem 1 above.

For manifolds with surface fundamental groups, we shall prove the following result.

THEOREM 5. Let $M$ and $N$ be closed connected orientable (smooth) 4-manifolds with $\Pi_{1}(M) \cong \Pi_{1}(N) \cong \Pi_{1}(F)$, where $F$ is a closed aspherical (orientable) surface. Then $M$ is simple homotopy equivalent to $N$ if and only if $M$ is TOP s-cobordant to $N$.

Theorem 5 together with the results proved in [7] imply the following consequence.

Corollary 6. Let $M$ be as above. Suppose further that $M$ is a spin manifold. Then $M$ is TOP s-cobordant to the connected sum of the product $F \times \mathbb{S}^{2}$ with a simplyconnected 4-manifold.

\section{Results from SURgery Theory}

The proofs of our results use surgery theory (see for example $[3,11,16,18,23]$ ) as corrected in [8]. To make the reading easier, we recall some definitions and results listed in the quoted papers. Note that the Whitehead group Wh $\left(\Pi_{1}\right)$ vanishes for $\Pi_{1} \cong *_{p} \mathbb{Z}$ or $\Pi_{1} \cong \Pi_{1}(F), F$ an aspherical surface, hence in our case "s-cobordant" is equivalent to "h-cobordant" (see [17]). 
Let $M^{n}$ be any closed connected orientable $(\mathrm{H}=$ TOP or PL) $n$-manifold with the fundamental group $\Pi_{1}=\Pi_{1}(M)$ and let $\xi^{k}$ be a linear bundle over $M$. Then $\Omega_{n}^{H}(M, \xi)$ denotes the set of bordism classes of normal maps $(X, f, b)$, where $X$ is a $(\mathrm{H}=\mathrm{TOP}$ or PL) $n$-manifold, $f: X \rightarrow M$ a map of degree one, $b: \nu_{X}^{k} \rightarrow \xi^{k}$ a linear bundle map covering $f$ and $\nu_{X}^{k}$ is the stable normal bundle of $X^{n} \rightarrow \mathbb{S}^{n+k}$, for $k$ sufficiently large with respect to $n$.

Let $\mathcal{N}_{n}^{H}(M)$ be the union of all $\Omega_{n}^{H}(M, \xi)$ over all $k$-plane bundles $\xi^{k}$ over $M$, modulo the additional equivalence relation that $\left(X_{0}, f_{0}, b_{0}\right) \in \Omega_{n}^{H}\left(M, \xi_{0}\right)$ is equivalent to $\left(X_{1}, f_{1}, b_{1}\right) \in \Omega_{n}^{H}\left(M, \xi_{1}\right)$ if and only if $\left(X_{0}, f_{0}, b_{0}\right)$ is normally (H=TOP or PL) cobordant to $\left(X_{1}, f_{1}, b_{1}\right)$, for some linear bundle automorphism $\xi_{1} \rightarrow \xi_{0}$. The elements of $\mathcal{N}_{n}^{H}(M)$ are called the (H=TOP or $\left.\mathrm{PL}\right)$ normal invariants of $M$. In the relative case, we include the condition that the normal map $(X, f, b) \in \mathcal{N}_{n}^{H}(M, \partial M)$, represented by $f:(X, \partial X) \rightarrow(M, \partial M)$, induces a simple homotopy equivalence when restricted to $\partial X$.

Let $\mathcal{S}_{n}^{H}(M)$ be the set of (H=TOP or PL) $s$-cobordism classes of orientation preserving simple homotopy equivalences $h: X \rightarrow M$, where $X$ is a compact (H) $n$ manifold.

Let us denote by $L_{n}\left(\Pi_{1}\right)$ the $n$-th Wall group of surgery obstructions for the problem of obtaining simple homotopy equivalences for orientable $n$-manifolds with the fundamental group $\mathrm{II}_{1}$.

Recall that if $h: X \rightarrow M$ represents an element of $\mathcal{S}_{n}^{H}(M)$, then there exists an obvious forgetful map $\eta_{n}^{H}: \mathcal{S}_{n}^{H}(M) \rightarrow \mathcal{N}_{n}^{H}(M)$ which associates to $(X, h)$ the class of $\left(X, h, h^{*}\right)$ in $\mathcal{N}_{n}^{H}(M)$, where $h^{*}$ is the obvious map on the stable normal bundles, induced by $h$.

Furthermore, there is a map $\sigma_{n}^{H}: \mathcal{N}_{n}^{H}(M) \rightarrow L_{n}\left(\Pi_{1}\right)$ which associates to any normal invariant $(X, f, b)$ the surgery obstruction (for details see [16] and [23]).

Finally, denote by $\omega_{n}^{H}: L_{n+1}\left(\Pi_{1}\right) \rightarrow \mathcal{S}_{n}^{H}(M)$ the map induced by the action of $L_{n+1}\left(\Pi_{1}\right)$ on $\mathcal{S}_{n}^{H}(M)$ (see for example [16]).

The following result is well-known (see $[11$, p.200]).

THEOREM 7 . (The surgery sequence).

Let $M^{n}$ be a closed connected orientable (H=TOP or $\left.P L\right)$-manifold with the fundamental group $\Pi_{1}$. Then the surgery sequence

$$
L_{n+1}\left(\Pi_{1}\right) \stackrel{\omega_{n}^{H}}{\longrightarrow} \mathcal{S}_{n}^{H}(M) \stackrel{\eta_{n}^{H}}{\longrightarrow} \mathcal{N}_{n}^{H}(M) \stackrel{\sigma_{n}^{H}}{\longrightarrow} L_{n}\left(\Pi_{1}\right)
$$

is exact if $n \geqslant 5$. If $n=4$, it is also exact provided $\Pi_{1}$ is an elementary amenable group.

For our classes of 4-manifolds we obtain a further result. 
TheOREM 8. Let $M$ be a closed connected orientable (H=TOP or PL) 4manifold. Suppose that $\Pi_{1}(M)$ is isomorphic to either $*_{p} \mathbb{Z}, p \geqslant 1$, or $\Pi_{1}\left(F_{g}\right)$, where $F_{g}$ is the closed orientable surface of genus $g \geqslant 1$. Then the surgery sequence

$$
\mathcal{N}_{5}^{\text {TOP }}(M) \stackrel{\sigma_{5}^{\text {TOP }}}{\longrightarrow} L_{5}\left(\Pi_{1}\right) \stackrel{\omega_{4}^{\text {TOP }}}{\longrightarrow} \mathcal{S}_{4}^{\text {TOP }}(M) \stackrel{\eta_{4}^{\text {TOP }}}{\longrightarrow} \mathcal{N}_{4}^{\text {TOP }}(M)
$$

is exact.

Proof: Let $k$ denote either of the integers $p$ or $2 g$. We shall prove that every element of $L_{5}\left(\Pi_{1}\right)$ is realisable by an element in $\Omega_{5}^{\text {TOP }}(M \times I, M \times \partial I), I=[0,1]$. Thus the result follows from Theorem 6.3 of [16] (second part of the statement which is correct; compare also with [8]).

Since $\Pi_{1}(M) \cong *_{k} \mathbb{Z}$ or $\cong \Pi_{1}\left(F_{g}\right)$, we have that $L_{5}\left(\Pi_{1}\right) \cong \oplus_{k} \mathbb{Z}$ by Theorem 16 of [4]. Because $M$ is orientable, any embedded 1-sphere $\tilde{f}: \mathbb{S}^{1} \rightarrow M$ has a trivial normal bundle, that is, $\tilde{f}$ extends to an embedding $f: \mathbb{S}^{1} \times D^{3} \rightarrow M$. Let $f_{1}, f_{2}, \ldots$, $f_{k}: \mathbb{S}^{1} \times D^{4} \rightarrow M \times I$ be disjoint embeddings such that

$$
\tilde{f}_{1}=\left.f_{1}\right|_{\$^{1} \times 0}, \quad \tilde{f}_{2}=\left.f_{2}\right|_{\mathbf{s}^{1} \times 0}, \ldots, \quad \tilde{f}_{k}=\left.f_{k}\right|_{\mathbf{s}^{1} \times 0}
$$

represent a set of generators of $\Pi_{1}(M \times I) \cong \Pi_{1}(M)$. This is always possible by the general position theorem. Let $N_{i}, i=1,2, \ldots, k$, be the TOP 5 -manifold obtained by deleting $f_{i}\left(\mathbb{S}^{1} \times \stackrel{\circ}{D^{4}}\right)$ from $M \times I$ and substituting $\left(\mathbb{S}^{1} \times\left\|E_{8}\right\|\right) \backslash\left(\mathbb{S}^{1} \times{\stackrel{\circ}{D^{4}}}^{4}\right)$ by an obvious identification of their boundaries. Here $\left\|E_{8}\right\|$ represents the simply connected TOP 4-manifold realising the form $E_{8}$ as constructed in [10].

Using an appropriate normal map

$$
\mathbb{S}^{1} \times\left\|E_{8}\right\| \rightarrow \mathbb{S}^{1} \times \mathbb{S}^{4}
$$

we obtain a normal map of degree one

$$
\xi_{i}: N_{i} \rightarrow M \times I=(M \times I) \backslash f_{i}\left(\mathbb{S}^{1} \times \stackrel{0}{D^{4}}\right) \cup_{\mathbb{S}^{1} \times \mathbb{S}^{3}} \mathbb{S}^{1} \times \mathbb{S}^{4} \backslash \mathbb{S}^{1} \times \stackrel{0}{D^{4}},
$$

hence $\left(N_{i}, \xi_{i}, \xi_{i}^{*}\right) \in \Omega_{5}^{\text {TOP }}\left(M \times I, M \times \partial I, \xi_{i}\right)$. Furthermore, the surgery obstruction $\sigma_{5}\left(N_{i}, \xi_{i}, \xi_{i}^{*}\right)$ is exactly the $i$-th generator of $L_{5}\left(\Pi_{1}\right) \cong \oplus_{k} \mathbb{Z}$. This completes the proof.

We can now apply Lemma 8 of [13] to obtain the following consequence.

Corollary 9. Let $M$ be as in Theorem 8. Then the map

$$
\eta_{4}^{\text {TOP }}: \mathcal{S}_{4}^{\text {TOP }}(M) \rightarrow \mathcal{N}_{4}^{\text {TOP }}(M)
$$


is injective.

Proof: It was proved in [13] that the surgery obstruction map

$$
\sigma_{5}^{\text {TOP }}: \mathcal{N}_{5}^{\text {TOP }}(M \times I, M \times \partial I) \rightarrow L_{5}\left(\Pi_{1}\right)
$$

is surjective. This guarantees the exactness of the sequence shown in Theorem 8. But if $\sigma_{5}^{\text {TOP }}$ is onto, then the map $\eta_{4}^{\text {TOP }}$ must be injective as claimed.

Now let $M^{4}$ be a closed connected orientable (smooth or PL) 4-manifold. Following [8], let us denote by $\mathrm{HE}_{\mathrm{Id}}(M)$ the set of homotopy classes of (simple) self-homotopy equivalences of $M$ which induce the identities on $\Pi_{1}$ and on $H_{*}$. Recall that any (simple) homotopy equivalence defines a normal invariant. This gives rise to a map

$$
n: \mathrm{HE}_{\mathrm{Id}}(M) \rightarrow \mathcal{N}_{4}^{\mathrm{PL}}(M) .
$$

The following result is based upon an argument of Wall (see [23, Theorem 16.6] and [16, Theorem 6.3]: first part of the statement) as corrected by Cochran-Habegger [8].

THEOREM 10. Let $M^{4}$ be a closed connected orientable (smooth or PL) 4manifold. Suppose that $H_{2}\left(\Pi_{1}(M) ; \mathbb{Z}_{2}\right) \cong 0$. Then the sequence

$$
\mathrm{HE}_{\mathrm{Id}}(M) \stackrel{n}{\longrightarrow} \mathcal{N}_{4}^{\mathrm{PL}}(M) \stackrel{\sigma_{4}^{\mathrm{PL}}}{\longrightarrow} L_{4}\left(\Pi_{1}(M)\right)
$$

is exact.

Proof: We first calculate the set of normal maps $\mathcal{N}_{4}^{H}(M)$ by the method of Sullivan (see [15] and [20]). There is a bijection between $\mathcal{N}_{4}^{H}(M), \mathrm{H}=\mathrm{TOP}$ or PL, and the group $[M, G / H]$ of the homotopy classes of maps $M \rightarrow G / H$ (see for example [16, Theorem 5.4]). Since $\Pi_{2}(G / \mathrm{TOP}) \cong \mathbb{Z}_{2}, \Pi_{3}(G / \mathrm{TOP}) \cong \Pi_{5}(G / \mathrm{TOP}) \cong 0$ and $\Pi_{4}(G / \mathrm{TOP}) \cong \mathbb{Z}$ with vanishing $k$-invariant in $H^{5}\left(K\left(\mathbb{Z}_{2}, 2\right)\right)$, the Postnikov resolution of $G /$ TOP gives a map $G /$ TOP $\rightarrow K\left(\mathbb{Z}_{2}, 2\right) \times K(\mathbb{Z}, 4)$ which is a 5 -equivalence, that is, we can assume that the 5 -skeleton of $G /$ TOP is the same as that of $K\left(\mathbb{Z}_{2}, 2\right) \times K(\mathbb{Z}, 4)$. Thus we have

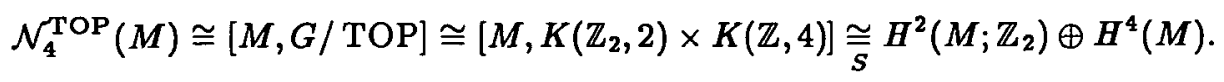

The injection $\mathcal{N}_{4}^{\mathrm{PL}}(M) \rightarrow \mathcal{N}_{4}^{\text {TOP }}(M)$ yields the isomorphisms (use the Wu formula)

$$
\begin{aligned}
& S: \mathcal{N}_{4}^{\mathrm{PL}}(M) \cong[M, G / \mathrm{PL}] \cong\left\{(a, b) \in H^{2}\left(M ; \mathbb{Z}_{2}\right) \oplus H^{4}(M): a^{2} \equiv b \bmod 2\right\} \\
& \cong w_{2}(M)^{\perp} \oplus H^{4}(M) \cong \operatorname{Ker} w_{2}(M) \oplus H^{4}(M) \cong \operatorname{Hom}\left(\operatorname{Ker} w_{2}(M), \mathbb{Z}_{2}\right) \oplus H^{4}(M),
\end{aligned}
$$

where $w_{2}(M)^{\perp}=\left\{a \in H^{2}\left(M ; \mathbb{Z}_{2}\right): a^{2} \equiv 0 \bmod 2\right\}$. 
The map $\mathcal{N}_{4}^{H}(M) \rightarrow H^{4}(M) \rightarrow L_{4}(1) \cong \mathbb{Z} \subset L_{4}\left(\Pi_{1}\right)$ is given by the surgery obstruction (see [23, p.237]). Thus we obtain $\operatorname{Ker} \sigma_{4}^{\mathrm{TOP}} \subset H^{2}\left(M ; \mathbb{Z}_{2}\right)$ and $\operatorname{Ker} \sigma_{4}^{\mathrm{PL}} \subset$ $\operatorname{Hom}\left(\operatorname{Ker} w_{2}(M), \mathbb{Z}_{2}\right)$. Now by $[21]$ we can represent a basis of $\operatorname{Ker} w_{2}(M)$ by characteristic 2-submanifolds $V_{i}^{2}$ of $M$. From our hypothesis, that $H_{2}\left(\Pi_{1} ; \mathbb{Z}_{2}\right) \cong 0$, we are going to prove that any assignment of elements of $\mathbb{Z}_{2}$ to the classes $\left[V_{i}^{2}\right] \in \operatorname{Ker} w_{2}(M)$ is induced by a self-homotopy equivalence in $\mathrm{HE}_{\mathrm{Id}}(M)$. In particular, we have $\operatorname{Ker} \sigma_{4}^{\mathrm{PL}}=w_{2}(M)^{\perp}=\operatorname{Hom}\left(\operatorname{Ker} w_{2}(M), \mathbb{Z}_{2}\right)$. Suppose $[V] \in \operatorname{Ker} w_{2}(M)$ is one of the previous characteristic submanifolds. The exact sequence

$$
\Pi_{2}(M) \rightarrow H_{2}\left(M ; \mathbb{Z}_{2}\right) \rightarrow H_{2}\left(\Pi_{1}(M) ; \mathbb{Z}_{2}\right) \cong 0
$$

implies that there exists a map $x: \mathbb{S}^{2} \rightarrow M$ which is sent to $[V]$ under the surjection $\Pi_{2}(M) \rightarrow H_{2}\left(M ; \mathbb{Z}_{2}\right)$. Choose an embedding $D^{4} \subset M$. If $D^{4}$ is shrunk to a point, then the result is homeomorphic to $M$. Shrink instead $\partial D^{4}$ to a point to give a map $c: M \rightarrow M \vee \mathbb{S}^{4}$. Now let $\eta: \mathbb{S}^{3} \rightarrow \mathbb{S}^{2}$ be the Hopf map, $\Sigma \eta: \mathbb{S}^{4} \rightarrow \mathbb{S}^{3}$ its suspension and $\eta^{2}: \mathbb{S}^{4} \rightarrow \mathbb{S}^{2}$ the composition $\eta^{2}=\eta \circ \Sigma \eta$. Let $f: M \rightarrow M$ be the composite map

$$
M \stackrel{c}{\longrightarrow} M \vee \mathbb{S}^{4} \stackrel{1 \vee \eta^{2}}{\longrightarrow} M \vee \mathbb{S}^{2} \stackrel{1 \vee x}{\longrightarrow} M .
$$

It is easy to see that $f$ induces the identities on $\Pi_{1}$ and on $H_{*}$, hence $f$ is a homotopy equivalence by Theorem 5.5 of [16]. To compute the splitting invariant of the map $f$ along $V$, we assume $V$ disjoint from $D^{4}$. Then $f^{-1}(V)=V \cup W$ with $W$ framed in $D^{4}$ and the splitting invariant is the Arf invariant of $W$. We may always assume that $x: \mathbb{S}^{2} \rightarrow M$ is an immersion which is transverse to $V$ in $M$, that is, $x\left(\mathbb{S}^{2}\right)$ meets $V$ transversely in $n_{V}$ points. Then $W$ is the union of $n_{V}$ preimages (torii) in $\mathbb{S}^{4}$ (top cell) of oriented points in $\mathbb{S}^{2}$ under the map $\eta^{2}: \mathbb{S}^{4} \rightarrow \mathbb{S}^{2}$. The associated quadratic form defined on $\operatorname{Ker}\left(f_{*}: H_{1}\left(f^{-1}(V)\right) \rightarrow H_{1}(V)\right)$ is the direct sum of its restrictions to $H_{1}(T)$ for each component torus $T$ so that it suffices to compute the appropriate Arf invariant for a single torus $T$. Since $T$ maps to a point in $V$, it follows by [8] that the quadratic form on $H_{1}(T)$ has Arf invariant $1+w_{2}([x])=1+w_{2}([V])=1$ as $[V] \in \operatorname{Ker} w_{2}(M)$. Hence each of the preimages in $W$ has Arf invariant one. Thus the required $f \in \mathrm{HE}_{\mathrm{Id}}(M)$ can be constructed if $[x] \in \Pi_{2}(M)$ is dual to a mod 2 cohomology class which assignes to each $V_{i}^{2}$ the given corresponding element of $\mathbb{Z}_{2}$. Because $\Pi_{2}(M) \rightarrow H_{2}\left(M ; \mathbb{Z}_{2}\right)$ is surjective such an $x$ exists. This proves the statement.

Proof of Theorem 1:

Let $h: N \rightarrow M$ be a simple homotopy equivalence. Since $H_{2}\left(\Pi_{1}(M) ; \mathbb{Z}_{2}\right)=$ $H_{2}\left(*_{p} \mathbb{Z} ; \mathbb{Z}_{2}\right) \cong 0$, there exists a simple self-homotopy equivalence $f \in \mathrm{HE}_{\mathrm{Id}}(M)$ such that $n(f)=n(h) \in \mathcal{N}_{4}^{\text {PL }}(M)$ by Theorem 10 . Hence it follows that $\eta_{4}^{\text {TOP }}(f)=$ 
$\eta_{4}^{\text {TOP }}(h) \in \mathcal{N}_{4}^{\text {TOP }}(M)$. By Corollary $9 f$ is TOP $s$-cobordant to $h$ in $\mathcal{S}_{4}^{\text {TOP }}(M)$, that is, $M$ is TOP $s$-cobordant to $N$. The second part of the statement follows in the same way by using Theorem 1 of [6].

We conclude the section with a related computation.

Proposition 11. Let $M^{4}$ be a closed connected orientable (TOP or PL) 4manifold with fundamental group $\Pi_{1} \cong *_{p} \mathbb{Z}, p \geqslant 1$. Then there is a bijection between $\mathcal{S}_{5}^{\text {TOP }}(M \times I, M \times \partial I)$ and $H^{1}\left(M ; \mathbb{Z}_{2}\right)$, hence the number of distinct topological 5manifolds homotopy equivalent to $(M \times I, M \times \partial I)$ is at most $2^{p}$.

Proof: Since the surgery obstruction map $\sigma_{5}^{\text {TOP }}$ is onto (see $[13$, Lemma 8$]$ ), the sequence

$$
\mathcal{S}_{5}^{\text {TOP }}(M \times I, M \times \partial I) \stackrel{\eta_{5}^{\text {TOP }}}{\longrightarrow} \mathcal{N}_{5}^{\text {TOP }}(M \times I, M \times \partial I) \stackrel{\sigma_{5}^{\text {TOP }}}{\longrightarrow} L_{5}\left(\Pi_{1}\right) \longrightarrow 0
$$

is exact (see [16, Theorem 5.11]). We are going to prove that $\eta_{5}^{\text {TOP }}$ is injective. Since $L_{6}\left(*_{p} \mathbb{Z}\right) \cong \mathbb{Z}_{2}$ (see [4, Theorem 1.6]), the map $L_{6}(1) \cong \mathbb{Z}_{2} \rightarrow L_{6}\left(*_{p} \mathbb{Z}\right) \cong \mathbb{Z}_{2}$ is an isomorphism, hence one can represent the nontrivial element of $L_{6}$ by a degree one normal map $\left(\mathbb{S}^{3} \times \mathbb{S}^{3}, f, b\right)$ with $f: \mathbb{S}^{3} \times \mathbb{S}^{3} \rightarrow \mathbb{S}^{6}$ (see [16] and [23]). Then the action of $L_{6}\left(*_{p} \mathbb{Z}\right) \cong \mathbb{Z}_{2}$ on $\mathcal{S}_{5}^{\text {TOP }}(M \times I, M \times \partial I)$ is defined by taking an element $h:(K, \partial K) \rightarrow(M \times I, M \times \partial I)$ in $\mathcal{S}_{5}^{\text {TOP }}(M \times I, M \times \partial I)$ and forming the connected sum in the interior $(h \times 1) \# f: K \times I \# \mathbb{S}^{3} \times \mathbb{S}^{3} \rightarrow M \times I^{2}=M \times I^{2} \# \mathbb{S}^{6}$. Using the additivity of surgery obstructions and the fact $\sigma_{6}(h \times 1)=0$, we have that $\sigma_{6}(h \times 1 \# f)=\sigma_{6}(f)$ so the action of $L_{6}\left(*_{p} \mathbb{Z}\right)$ on $\mathcal{S}_{5}^{\mathrm{TOP}}(M \times I, M \times \partial I)$ is trivial. Thus $\eta_{5}^{\text {TOP }}$ is injective as claimed. By Sullivan [20], there is a bijection between $\mathcal{N}_{5}^{\text {TOP }}(M \times I, M \times \partial I)$ and the group $[(M \times I, M \times \partial I),(G / \mathrm{TOP}, *)]$.

Hence we have

$$
\begin{aligned}
& \mathcal{N}_{5}^{\mathrm{TOP}}(M \times I, M \times \partial I) \cong\left[M \times I / M \times \partial I, K\left(\mathbb{Z}_{2}, 2\right) \times K(\mathbb{Z}, 4)\right] \\
& \cong H^{2}\left(\Sigma M ; \mathbb{Z}_{2}\right) \oplus H^{4}(\Sigma M) \cong H^{1}\left(M ; \mathbb{Z}_{2}\right) \oplus H^{3}(M) \cong \oplus_{p} \mathbb{Z}_{2} \oplus \oplus_{p} \mathbb{Z}
\end{aligned}
$$

where $\Sigma M$ denotes the suspension of $M$. Putting all these facts together we have the exact sequence

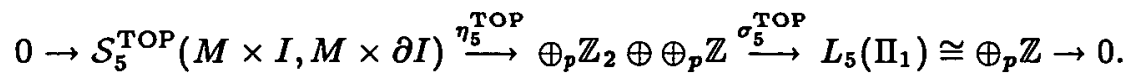

Thus we obtain that

$$
\operatorname{Ker} \sigma_{5}^{\mathrm{TOP}} \cong \operatorname{Im} \eta_{5}^{\mathrm{TOP}} \cong \oplus_{p} \mathbb{Z}_{2} \cong H^{1}\left(M ; \mathbb{Z}_{2}\right) \cong \mathcal{S}_{5}^{\mathrm{TOP}}(M \times I, M \times \partial I)
$$

as required. 


\section{Free groups}

In this section we shall present a simple alternative proof of Theorem 1 which is essentially based upon some recent results due to Cochran and Habegger (see [8]).

Let $M^{4}$ be a closed connected orientable (smooth or PL) 4-manifold with fundamental group $\Pi_{1}(M) \cong *_{p} \mathbb{Z}, p \geqslant 1$. Choose embeddings $\varphi_{i}: \mathbb{S}^{1} \times D^{3} \rightarrow M$, $i=1,2, \ldots, p$, such that

$$
\left.\varphi_{1}\right|_{\$^{1} \times 0},\left.\varphi_{2}\right|_{\mathbb{S}^{1} \times 0}, \ldots,\left.\varphi_{p}\right|_{\mathbb{S}^{1} \times 0}
$$

represent a set of generators of $\Pi_{1}(M)$.

Let

$$
M_{0}=M \backslash \bigcup_{i=1}^{p} \varphi_{i}\left(\mathbb{S}^{1} \times \stackrel{\circ}{D}^{3}\right)
$$

and

$$
M^{\prime}=M_{0} \cup \bigcup_{i=1}^{p}\left(D^{2} \times \mathbb{S}^{2}\right) \text {. }
$$

The manifold $M^{\prime}$ is a simply-connected closed 4 -manifold obtained by surgeries killing the fundamental group $\Pi_{1}(M)$.

Moreover, the canonical inclusions $M_{0} \rightarrow M$ and $M_{0} \rightarrow M^{\prime}$ induce isomorphisms

$$
H_{2}(M) \longleftarrow H_{2}\left(M_{0}\right) \underset{\cong}{\simeq} H_{2}\left(M^{\prime}\right)
$$

which respect the integral intersection forms (see for example [5]).

By Sullivan's results (see [20]) there are natural isomorphisms

$$
\begin{gathered}
S: \mathcal{N}_{4}^{\mathrm{PL}}(M) \cong\left\{(a, b) \in H^{2}\left(M ; \mathbb{Z}_{2}\right) \times H^{4}(M ; \mathbb{Z}): a^{2} \equiv b \bmod 2\right\}, \\
S^{\prime}: \mathcal{N}_{4}^{\mathrm{PL}}\left(M^{\prime}\right) \cong\left\{\left(a^{\prime}, b^{\prime}\right) \in H^{2}\left(M^{\prime} ; \mathbb{Z}_{2}\right) \times H^{4}\left(M^{\prime} ; \mathbb{Z}\right):\left(a^{\prime}\right)^{2} \equiv b^{\prime} \bmod 2\right\},
\end{gathered}
$$

$S_{0}: \mathcal{N}_{4}^{\mathrm{PL}}\left(M_{0}, \partial M_{0}\right) \cong\left\{\left(a_{0}, b_{0}\right) \in H^{2}\left(M_{0}, \partial M_{0} ; \mathbb{Z}_{2}\right) \times H^{4}\left(M_{0}, \partial M_{0} ; \mathbb{Z}\right): a_{0}^{2} \equiv b_{0} \bmod 2\right\}$.

Recall that any homotopy equivalence of a manifold defines a normal invariant. This gives rise to maps

$$
\begin{aligned}
n: H E_{\mathrm{Id}}(M) & \rightarrow \mathcal{N}_{4}^{\mathrm{PL}}(M), \\
n^{\prime}: H E_{\mathrm{Id}}\left(M^{\prime}\right) & \rightarrow \mathcal{N}_{4}^{\mathrm{PL}}\left(M^{\prime}\right), \\
n_{0}: H E_{\mathrm{Id}}\left(M_{0}, \partial M_{0}\right) & \rightarrow \mathcal{N}_{4}^{\mathrm{PL}}\left(M_{0}, \partial M_{0}\right)
\end{aligned}
$$

where $H E_{\mathrm{Id}}\left(M_{0}, \partial M_{0}\right)=\left\{f \in H E_{\mathrm{Id}}\left(M_{0}\right):\left.f\right|_{\theta M_{0}}=\mathrm{Id}\right\}$ 
Furthermore, there are well-known maps

$$
\begin{aligned}
& \Pi_{2}(M) \otimes \Pi_{4}\left(\mathbb{S}^{2}\right) \stackrel{N}{\longrightarrow} \Pi_{4}(M) \stackrel{\tau}{\rightarrow} H E_{\mathrm{Id}}(M), \\
& \Pi_{2}\left(M^{\prime}\right) \otimes \Pi_{4}\left(\mathbb{S}^{2}\right) \stackrel{N^{\prime}}{\longrightarrow} \Pi_{4}\left(M^{\prime}\right) \stackrel{\tau^{\prime}}{\longrightarrow} H E_{\mathrm{Id}}\left(M^{\prime}\right), \\
& \Pi_{2}\left(M_{0}\right) \otimes \Pi_{4}\left(\mathbb{S}^{2}\right) \stackrel{N_{0}}{\longrightarrow} \Pi_{4}\left(M_{0}\right) \stackrel{\tau_{0}}{\longrightarrow} H E_{\mathrm{Id}}\left(M_{0}, \partial M_{0}\right)
\end{aligned}
$$

defined as follows. For any $[x] \in \Pi_{2}(M), x: \mathbb{S}^{2} \rightarrow M$, let

$$
N([x] \otimes 1)=[x \circ \eta \circ \Sigma \eta],
$$

where $\eta: \mathbb{S}^{3} \rightarrow \mathbb{S}^{2}$ is the Hopf map and $\Sigma \eta: \mathbb{S}^{4} \rightarrow \mathbb{S}^{3}$ is its suspension. The map $\tau$ is obtained by "pinching off" a 4-sphere from a 4-cell $B$ which we choose in Int $M_{0}$, that is, if $[y] \in \Pi_{4}(M), y: \mathbb{S}^{4} \rightarrow M$, then

$$
\tau([y]): M \longrightarrow M \vee \mathbb{S}^{4} \stackrel{\text { Id } \vee y}{\longrightarrow} M
$$

(see $[\mathbf{8}]$ or $[\mathbf{1 8}])$.

Let us denote by

$$
\begin{aligned}
\sigma & : \mathcal{N}_{4}^{\mathrm{PL}}(M) \rightarrow L_{4}\left(\Pi_{1}(M)\right), \\
\sigma^{\prime} & : \mathcal{N}_{4}^{\mathrm{PL}}\left(M^{\prime}\right) \rightarrow L_{4}(1) \\
\sigma_{0} & : \mathcal{N}_{4}^{\mathrm{PL}}\left(M_{0}, \partial M_{0}\right) \rightarrow L_{4}\left(\Pi_{1}\left(M_{0}\right)\right)
\end{aligned}
$$

the surgery obstruction maps. By [4] we have

$$
L_{4}\left(\Pi_{1}(M)\right) \cong L_{4}(1) \cong L_{4}\left(\Pi_{1}\left(M_{0}\right)\right) \cong \mathbb{Z} .
$$

The isomorphisms are induced by either $1 \rightarrow *_{p} \mathbb{Z}$ or $*_{p} \mathbb{Z} \rightarrow 1$. Since $\sigma, \sigma^{\prime}$ and $\sigma_{0}$ detect the "top-splitting invariant", one obtains

$$
\begin{aligned}
\operatorname{Ker} \sigma & =\left\{a \in H^{2}\left(M ; \mathbb{Z}_{2}\right): a^{2}=0\right\}=w_{2}(M)^{\perp}, \\
\operatorname{Ker} \sigma^{\prime} & =\left\{a^{\prime} \in H^{2}\left(M^{\prime} ; \mathbb{Z}_{2}\right):\left(a^{\prime}\right)^{2}=0\right\}=w_{2}\left(M^{\prime}\right)^{\perp}, \\
\operatorname{Ker} \sigma_{0} & =\left\{a_{0} \in H^{2}\left(M_{0}, \partial M_{0} ; \mathbb{Z}_{2}\right):\left(a_{0}\right)^{2}=0\right\}=w_{2}\left(M_{0}\right)^{\perp},
\end{aligned}
$$

as shown in the proof of Theorem 10 .

Let us consider the following diagram

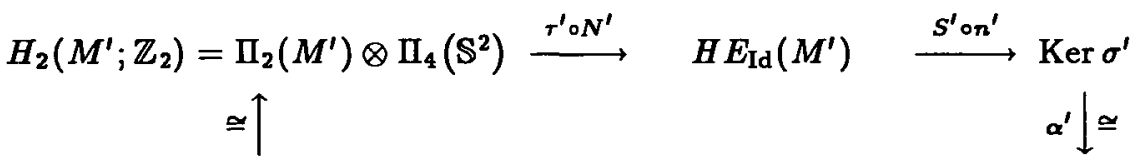

$$
\begin{aligned}
& H_{2}\left(M_{0} ; \mathbb{Z}_{2}\right) \subset \Pi_{2}\left(M_{0}\right) \otimes \Pi_{4}\left(\mathbb{S}^{2}\right) \stackrel{\tau_{0} \circ N_{0}}{\longrightarrow} H E_{\mathrm{Id}}\left(M_{0}, \partial M_{0}\right) \stackrel{S_{0} \circ n_{0}}{\longrightarrow} \operatorname{Ker} \sigma_{0} \\
& \simeq \downarrow \quad \alpha \uparrow \cong \\
& H_{2}\left(M ; \mathbb{Z}_{2}\right) \subset \Pi_{2}(M) \otimes \Pi_{4}\left(\mathbb{S}^{2}\right) \stackrel{\text { roN }}{\longrightarrow} \quad H E_{\mathrm{Id}}(M) \stackrel{\text { Son }}{\longrightarrow} \operatorname{Ker} \sigma
\end{aligned}
$$


where $\alpha: \operatorname{ker} \sigma \rightarrow \operatorname{Ker} \sigma_{0}$ and $\alpha^{\prime}: \operatorname{Ker} \sigma^{\prime} \rightarrow \operatorname{Ker} \sigma_{0}$ are the isomorphisms induced by

$$
H^{2}\left(M_{0}, \partial M_{0} ; \mathbb{Z}_{2}\right) \underset{\cong}{\cong} H^{2}\left(M_{0} ; \mathbb{Z}_{2}\right)
$$

and

$$
H^{2}\left(M ; \mathbb{Z}_{2}\right) \underset{\cong}{\simeq} H^{2}\left(M_{0} ; \mathbb{Z}_{2}\right) \stackrel{\cong}{\simeq} H^{2}\left(M^{\prime} ; \mathbb{Z}_{2}\right)
$$

as $w_{2}(:)$ is natural. It can be easily seen that the above diagram commutes. One of the main result of $[8]$ is that the maps $S^{\prime} \circ n^{\prime}$ and $\tau^{\prime} \circ N^{\prime}$ are bijective (see Theorem 5.2, Section 5).

\section{ProOF OF THEOREM 1:}

Let $h: N \rightarrow M$ be a simple homotopy equivalence, hence $n(h) \in \operatorname{Ker} \sigma$. By the above diagram and [8] there exists a homotopy equivalence $f: M \rightarrow M$ such that $n(f)=n(h)$.

Let

$$
\left(W^{5}, N, M\right) \stackrel{\xi}{\longrightarrow}(M \times I, M \times \partial I)
$$

be a normal TOP cobordism between $f$ and $h(I=[0,1])$. The proof now follows from the following lemma.

LEMMA 12. If $x \in L_{5}\left(\Pi_{1}(M \times I)\right) \cong \oplus_{p} \mathbb{Z}$ (see [4]), then there exists a normal TOP cobordism

$$
\eta:\left(V^{5}, \partial V=M \cup M\right) \rightarrow(M \times I, M \times \partial I)
$$

such that $\left.\eta\right|_{\partial V}=f \cup f$ and $\sigma(\eta)=x$.

If we take $y=-x=-\sigma(\eta)$, then the surgery obstruction of the composed normal map

$$
\xi \cup \eta:(W \cup V, N \cup M) \rightarrow(M \times[0,2], M \times \partial[0,2])
$$

is zero, hence by surgery one obtains an s-cobordism between $f$ and $h$ in $\mathcal{S}_{4}^{\text {TOP }}(M)$. Thus the manifolds $N$ and $M$ are TOP s-cobordant as claimed.

ProOF OF LEMMA 12 :

The proof proceeds in the usual way (see also Theorem 8). We start with the homotopy equivalence

$$
f \times \mathrm{Id}:(M \times I, M \times \partial I) \rightarrow(M \times I, M \times \partial I) .
$$

Since $f \in H E_{\mathrm{Id}}(M)$, we can assume that $f$ is the identity on each $\varphi_{i}\left(\mathbb{S}^{1} \times D^{4}\right)$, $i=1,2, \ldots, p$, which represent the generators of $\Pi_{1}(M)$.

Let $N_{i}, i=1,2, \ldots, p$, be the topological 5 -manifold obtained by deleting $\varphi_{i}\left(\mathrm{~S}^{1} \times \stackrel{\circ}{D^{4}}\right)$ from $M \times I$ and substituting $\left(\mathrm{S}^{1} \times\left\|E_{8}\right\|\right) \backslash\left(\mathrm{S}^{1} \times \stackrel{\circ}{D^{4}}\right)$ by an obvious identification of their boundaries (compare also with the proof of Theorem 8 ). 
Because $f$ is the identity on each $\varphi_{i}\left(\mathbb{S}^{1} \times D^{4}\right)$, using an appropriate normal map $\mathbb{S}^{1} \times\left\|E_{8}\right\| \rightarrow \mathbb{S}^{1} \times \mathbb{S}^{4}$, we obtain a normal TOP cobordism

$$
\xi_{i}: N_{i} \rightarrow M \times I=(M \times I) \backslash \varphi_{i}\left(\mathbb{S}^{1} \times \stackrel{\circ}{D}^{4}\right) \bigcup_{\mathbf{S}^{1} \times \mathbb{S}^{3}}\left(\mathbb{S}^{1} \times \mathbb{S}^{4} \backslash \mathbb{S}^{1} \times \stackrel{\circ}{D}^{4}\right) .
$$

such that $\left.\xi\right|_{\partial N_{i}}=f \cup f$ and the surgery obstruction $\sigma\left(\xi_{i}\right)$ is exactly the $i$-th generator of $L_{5}\left(\Pi_{1}\right)=\oplus_{p} Z$. This proves the lemma.

EXAMPLE. Suppose $p=1$, that is, $\Pi_{1}(M) \cong \mathbb{Z}, \lambda$ is an intersection form on a finitely generated free $\mathbb{Z}[\mathbb{Z}]$-module, $k \in \mathbb{Z}_{2}$ and if $\lambda$ is even, then we assume $k \equiv(\operatorname{signature} \lambda) / 8(\bmod 2)$. Then there is an oriented closed manifold $M_{\lambda}$ with $\Pi_{1} \cong \mathbb{Z}$, intersection form $\lambda$ and Kirby-Siebenmann invariant $k$ (see $[15, p .113]$ ). Hence the connected sum

$$
M=M_{\lambda_{1}} \# \ldots \# M_{\lambda_{p}} \# M^{\prime}
$$

(where $M^{\prime}$ is a simply connected manifold) has $\Pi_{1} \simeq *_{p} \mathbb{Z}$ and intersection form

$$
\lambda_{M}=\lambda_{1} \oplus \cdots \oplus \lambda_{p} \oplus \lambda_{M^{\prime}}
$$

on the $\Lambda$-module $\Pi_{2}(M, \Lambda), \Lambda=\mathbb{Z}\left[*_{p} \mathbb{Z}\right]$.

\section{Surface Groups}

In this section we shall prove Theorem 5 . The statement follows in the same way as in section 2 together with the following result.

THEOREM 13. Let $M$ be a closed connected orientable (smooth) 4-manifold with $\Pi_{1}(M) \cong \Pi_{1}(F)$, where $F$ is a closed aspherical (orientable) surface. Then the sequence

$$
\mathrm{HE}_{\mathrm{Id}}(M) \stackrel{n}{\longrightarrow} \mathcal{N}_{4}^{\mathrm{PL}}(M) \stackrel{\sigma_{4}^{\mathrm{PL}}}{\longrightarrow} L_{4}\left(\Pi_{1}(M)\right) \cong \mathbb{Z}_{2} \oplus \mathbb{Z}
$$

is exact.

ProOF: As proved in $[13$, p.279], we have

$$
\begin{aligned}
\Pi_{2}(M) \cong H_{2}(M ; \Lambda) & \cong \operatorname{Ext}_{\Lambda}^{2}\left(H_{0}(M ; \Lambda), \Lambda\right) \oplus \operatorname{Ext}_{\Lambda}^{0}\left(H_{2}(M ; \Lambda), \Lambda\right) \\
& \cong H^{2}(F) \oplus \operatorname{Ext}_{\Lambda}^{0}\left(H_{2}(M ; \Lambda), \Lambda\right),
\end{aligned}
$$

where $Q=\operatorname{Ext}_{\Lambda}^{0}\left(H_{2}(M ; \Lambda), \Lambda\right)$ is stably $\Lambda$-free. Using the universal coefficient spectral sequence

$$
\operatorname{Tor}_{p}^{\Lambda}\left(H_{q}(M ; \Lambda), \mathbb{Z}\right) \Longrightarrow H_{p+q}(M ; \mathbb{Z})
$$


we obtain

$$
\begin{aligned}
H_{2}(M ; \mathbb{Z}) & \cong \operatorname{Tor}_{0}^{\Lambda}\left(H_{2}(M ; \Lambda), \mathbb{Z}\right) \oplus \operatorname{Tor}_{2}^{\Lambda}\left(H_{0}(M ; \Lambda), \mathbb{Z}\right) \\
& \cong H_{2}(M ; \Lambda) \otimes_{\Lambda} \mathbb{Z} \oplus H_{2}\left(\Pi_{1} ; \mathbb{Z}\right) \cong \Pi_{2}(M) \otimes_{\Lambda} \mathbb{Z} \oplus H_{2}(F ; \mathbb{Z}),
\end{aligned}
$$

hence

$$
H^{2}\left(M ; \mathbb{Z}_{2}\right) \cong \Pi_{2}(M) \otimes_{\Lambda} \mathbb{Z}_{2} \oplus H_{2}\left(F ; \mathbb{Z}_{2}\right) \cong \Pi_{2}(M) \otimes_{\Lambda} \mathbb{Z}_{2} \oplus \mathbb{Z}_{2}
$$

It follows that

$$
\mathcal{N}_{4}^{\mathrm{TOP}}(M) \cong[M, G / \mathrm{TOP}] \cong \Pi_{2}(M) \otimes_{\Lambda} \mathbb{Z}_{2} \oplus \mathbb{Z}_{2} \oplus \mathbb{Z} .
$$

Since the surgery obstruction map $\sigma_{4}^{\text {TOP }}: \mathcal{N}_{4}^{\text {TOP }}(M) \rightarrow L_{4}\left(\Pi_{1}\right) \cong \mathbb{Z}_{2} \oplus \mathbb{Z}$ (see [4]) is onto (see [13, Lemma 8]), we have that $\operatorname{Ker} \sigma_{4}^{\text {TOP }} \cong \Pi_{2}(M) \otimes_{\Lambda} \mathbb{Z}_{2}$, that is, any element of $\operatorname{Ker} \sigma_{4}^{\text {TOP }}$ can be realised by a map $\mathbb{S}^{2} \rightarrow M$. Let $j$ denote the injection $\mathcal{N}_{4}^{\mathrm{PL}}(M) \rightarrow \mathcal{N}_{4}^{\mathrm{TOP}}(M)$. Since $\sigma_{4}^{\mathrm{PL}}=\sigma_{4}^{\mathrm{TOP}} \circ j$, we have that

$$
j\left(\operatorname{Ker} \sigma_{4}^{\mathrm{PL}}\right) \subset \operatorname{Ker} \sigma_{4}^{\mathrm{TOP}},
$$

hence $j\left(\operatorname{Ker} \sigma_{4}^{\mathrm{PL}}\right) \subset \Pi_{2}(M) \otimes_{\Lambda} \mathbb{Z}_{2}$. Now the proof can be completed as shown in Theorem 10 since any element of $\operatorname{Ker} \sigma_{4}^{\mathrm{PL}}$ can be realised by spherical maps. Indeed, the exact sequence

$$
\Pi_{2}(M) \rightarrow H_{2}\left(M ; \mathbb{Z}_{2}\right) \cong \Pi_{2}(M) \otimes_{\Lambda} \mathbb{Z}_{2} \oplus H_{2}\left(F ; \mathbb{Z}_{2}\right) \rightarrow H_{2}\left(\Pi_{1} ; \mathbb{Z}_{2}\right) \cong H_{2}\left(F ; \mathbb{Z}_{2}\right) \rightarrow 0
$$

shows that $\Pi_{2}(M)$ covers $\Pi_{2}(M) \otimes_{\Lambda} \mathbb{Z}_{2}$, as requested.

\section{REFERENCES}

[1] S. Bauer, The Homotopy type of a 4-manifold with finite fundamental group, Lecture Notes in Mathematics 1361 (Springer-Verlag, Berlin, Heidelberg, New York, 1988).

[2] H.J. Baues, Combinatorial homotopy and 4-dimensional complexes (Walter de Gruyter, Berlin, New York, 1991).

[3] S.E. Cappell and J. L. Shaneson, 'On four-dimensional surgery and applications', Comment. Math. Helv. 46 (1971), 500-528.

[4] S.E. Cappell, 'Mayer-Vietoris sequences in Hermitian K-theory', in Proc. Conf. Battelle Memorial Inst., Seattle, Wash., 1972, (Springer-Verlag, Berlin, Heidelberg, New York, 1973), pp. 478-512.

[5] A. Cavicchioli and F. Hegenbarth, 'On the intersection forms of closed 4-manifolds', Publ. Mat. 36 (1992), 73-83.

[6] A. Cavicchioli and F. Hegenbarth, 'On 4-manifolds with free fundamental group', Forum Math. 6 (1994), 415-429. 
[7] A. Cavicchioli, F. Hegenbarth and D. Repovš, 'Four-manifolds with surface fundamental groups' (to appear).

[8] T.D. Cochran and N. Habegger, 'On the homotopy theory of simply-connected four manifolds', Topology 29 (1990), 419-440.

[9] S.K. Donaldson, 'The orientation of Yang-Mills moduli spaces and 4-manifold topology', J. Differential Geom. 26 (1987), 397-428.

[10] M.H. Freedman, 'The topology of 4-manifolds', J. Differential Geom. 17 (1982), 357-453.

[11] M.H. Freedman and F. Quinn, Topology of 4-manifolds (Princeton Univ. Press, Princeton, New Jersey, 1990).

[12] I. Hambleton and M. Kreck, 'On the classification of topological 4-manifolds with finite fundamental group', Math. Ann. 280 (1988), 85-104.

[13] J.A. Hillman, 'On 4-manifolds homotopy equivalent to surface bundles over surfaces', Topology Appl. 40 (1991), 275-286.

[14] J.A. Hillman, 'Free products and 4-dimensional connected sums' (to appear).

[15] R.C. Kirby and L.C. Siebenmann, Foundational essays on topological manifolds, smoothings and triangulations, Ann. of Math. Studies 88 (Princeton Univ. Press, Princeton, New Jersey, 1977).

[16] R. Mandelbaum, 'Four-dimensional topology: an introduction', Bull. Amer. Math. Soc. 2 (1980), 1-159.

[17] J. Milnor, 'Whitehead torsion', Bull. Amer. Math. Soc. 72 (1966), 358-426.

[18]' J.L. Shaneson, 'Non-simply connected surgery and some results in low dimension topology', Comment. Math. Helv. 45 (1970), 333-352.

[19] J.L. Shaneson, 'On non-simply connected manifolds', in Proc. Sympos. Pure Math. 22 (Amer. Math. Soc., Providence, R.I., 1970), pp. 221-229.

[20] D. Sullivan, 'Triangulating and smoothing homotopy equivalences and homeomorphisms', in Geometric Topology Sem. Notes (Princeton Univ. Press, Princeton, New Jersey, 1967).

[21] R. Thom, 'Quelques propriétés globales des variétés', Comment. Math. Helv. 28 (1954), 17-86.

[22] C.T.C. Wall, 'On simply connected 4-manifolds', J. London Math. Soc. 39 (1964), 141-149.

[23] C.T.C. Wall, Surgery on Compact Manifolds (Academic Press, London, New York, 1970).

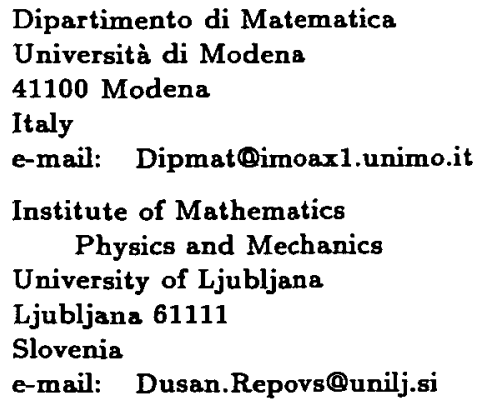

\author{
Dipartimento di Matematica \\ Università di Milano \\ 20133 Milano \\ Italy \\ e-mail: Dipmat@imiucca.csi.unimi.it
}

